\title{
Maximum displacement variability of stochastic structures subject to deterministic earthquake loading
}

\author{
L.L. Graham \\ Department of Civil Engineering, University of Virginia, Charlottesville, VA 22903-2442, USA
}

Received 8 August 1998

Revised 25 November 1998

\begin{abstract}
The variability of the maximum response displacement of random frame structures under deterministic earthquake loading are examined in this paper using stochastic finite element techniques. The elastic modulus and the mass density are assumed to be described by cross-correlated stochastic fields. Specifically, a variability response function formulation is used for this problem, which allows for calculation of spectral-distribution-free upper bounds of the maximum displacement variance. Further, under the assumption of prespecified correlation functions describing the spatial variation of the material properties, variability response functions are used to calculate the corresponding maximum displacement variance. Two numerical examples are provided to demonstrate the methodology. Results show that randomness in the material properties can lead to significant uncertainty in the maximum response displacement.
\end{abstract}

\section{Introduction}

For the design of structures, it is often sufficient to evaluate the maximum response of the structure to a given loading. To analyze the maximum structural response under earthquake loading, a design response spectrum is generally used. While this measure of the maximum response usually accounts for randomness in the loading (the earthquake), it does not account for randomness in the material/geometric properties of the structure. In this paper, variability response functions (see Deodatis and Graham [7] for their definition) are used to analyze the random maximum deflection of a structure with a stochastically varying elastic modulus and mass density that is subjected to a deterministic (design) earthquake loading.

This variability response function analysis is performed in the context of the random eigenvalue problem, which first came under scrutiny over twenty five years ago. At this time, analytical solutions and direct simulation of stochastic fields were performed for relatively simple structural systems (see, e.g., Boyce [2], Boyce and Xia [3], Collins and Thomson [5], Fox and Kapoor [8], Grigoriu [10], Hasselman and Hart [11], Hoshiya and Shah [12], Huang [13], Ibrahim [14], Iyengar and Manohar [15], Manohar and Iyengar [17], Purkert and Vom Scheidt [20], Shinozuka and Astill [23], Soong and Bogdanoff [24], Vaicaitis [28]). The emergence of stochastic finite element methods has provided a means of performing stochastic analyses of more complicated structural systems, leading to a renewed interest in the random eigenvalue problem (Koyluoglu [16], Spanos and Zeldin [25], Nagashima and Tsutsumi [18], Nakagiri et al. [19], Ramu and Ganesan [21], Ramu et al. [22], and Zhang and Chen [29]). In most of the work in this area, it was assumed that the stochastic material properties are represented by independent stochastic fields. In general, however, it is expected that there may be some cross-correlation between material properties such as the mass density and the elastic modulus. Recently Graham and Deodatis [9] analyzed the eigenvalue variability for beam-column and plate structures with the mass density and the elastic modulus represented by cross-correlated stochastic fields using variability response functions. This paper uses the techniques presented in that work to evaluate the maximum displacement variability of structures with random eigenvalues. 
Describing the elastic modulus and mass density as stochastic fields, the weighted integral method (Deodatis [6], Takada [26]) is used to represent the stochastic mass and stiffness matrices as linear combinations of random variables. Variability response functions for the maximum deflection are then formulated, allowing estimates to be made of the spectral-distribution-free upper bounds on the maximum deflection variability. These bounds are most interesting for real engineering applications, because very little probabilistic information is generally available about the material properties. However, assuming that the spectral density functions describing these properties are known or can be assumed, variability response functions can be applied to calculate the corresponding maximum deflection variability. In order to demonstrate these capabilities, numerical examples will be provided for two reinforced concrete frames.

\section{Maximum deflection variability}

The 2-node, 6 degree-of-freedom beam/column finite element (two displacements and one rotation at each node) is used in this paper. For the beam/column finite elements considered here, the elastic modulus $E$ and mass density $\rho$ are assumed to vary randomly along the length of the element as:

$$
\begin{gathered}
E(x)=E_{0}[1+f(x)], \quad-1<f(x), \\
\rho(x)=\rho_{0}[1+g(x)], \quad-1<g(x),
\end{gathered}
$$

where $E_{0}$ and $\rho_{0}$ are the mean values of the elastic modulus and mass density, respectively, and $f(x)$ and $g(x)$ are zero-mean homogeneous stochastic fields, which are assumed to be cross-correlated. Substituting the expressions for the randomly varying elastic modulus and mass density into the standard finite element formulations, the stochastic element stiffness and mass matrices were derived by Deodatis and Graham [7] as:

$$
\begin{aligned}
& \mathbf{K}^{(e)}=\mathbf{K}_{0}^{(e)}+\Delta \mathbf{K}_{1}^{(e)} \cdot X_{1}^{(e)}+\Delta \mathbf{K}_{2}^{(e)} \cdot X_{2}^{(e)}+\Delta \mathbf{K}_{3}^{(e)} \cdot X_{3}^{(e)}, \\
& \mathbf{M}^{(e)}=\mathbf{M}_{0}^{(e)}+\Delta \mathbf{M}_{1}^{(e)} \cdot Y_{1}^{(e)}+\cdots+\Delta \mathbf{M}_{7}^{(e)} \cdot Y_{7}^{(e)},
\end{aligned}
$$

where $\mathbf{K}_{0}^{(e)}$ and $\mathbf{M}_{0}^{(e)}$ are the deterministic parts of the element stiffness and mass matrix, respectively, which are obtained using the mean values of the elastic modulus and mass density:

$$
\mathbf{K}_{0}^{(e)}=E_{0}\left[\begin{array}{cccccc}
\frac{A}{L} & 0 & 0 & -\frac{A}{L} & 0 & 0 \\
0 & \frac{12 I}{L^{3}} & \frac{6 I}{L^{2}} & 0 & -\frac{12 I}{L^{3}} & \frac{6 I}{L^{2}} \\
0 & \frac{6 I}{L^{2}} & 4 \frac{I}{L} & 0 & -\frac{6 I}{L^{2}} & 2 \frac{I}{L} \\
-\frac{A}{L} & 0 & 0 & \frac{A}{L} & 0 & 0 \\
0 & -\frac{12 I}{L^{3}} & -\frac{6 I}{L^{2}} & 0 & \frac{12 I}{L^{3}} & -\frac{6 I}{L^{2}} \\
0 & \frac{6 I}{L^{2}} & 2 \frac{I}{L} & 0 & -\frac{6 I}{L^{2}} & 4 \frac{I}{L}
\end{array}\right],
$$




$$
\mathbf{M}_{0}^{(e)}=\frac{\rho_{0} L}{4}\left[\begin{array}{cccccc}
\frac{4}{3} & 0 & 0 & \frac{2}{3} & 0 & 0 \\
0 & \frac{52}{35} & \frac{22 L}{105} & 0 & \frac{18}{35} & -\frac{13 L}{105} \\
0 & \frac{22 L}{105} & \frac{4 L^{2}}{105} & 0 & \frac{13 L}{105} & -\frac{L^{2}}{35} \\
\frac{2}{3} & 0 & 0 & \frac{4}{3} & 0 & 0 \\
0 & \frac{18}{35} & \frac{13 L}{105} & 0 & \frac{52}{35} & -\frac{22 L}{105} \\
0 & -\frac{13 L}{105} & -\frac{L^{2}}{35} & 0 & -\frac{22 L}{105} & \frac{4 L^{2}}{105}
\end{array}\right],
$$

$\Delta \mathbf{K}_{k}^{(e)}, k=1,2,3$, are deterministic matrices:

$$
\begin{gathered}
\Delta \mathbf{K}_{1}^{(e)}=\frac{E_{0}}{2 L}\left[\begin{array}{cccccc}
A & 0 & 0 & -A & 0 & 0 \\
0 & 0 & 0 & 0 & 0 & 0 \\
0 & 0 & I & 0 & 0 & -I \\
-A & 0 & 0 & A & 0 & 0 \\
0 & 0 & 0 & 0 & 0 & 0 \\
0 & 0 & -I & 0 & 0 & I
\end{array}\right], \\
\boldsymbol{\Delta K}_{2}^{(e)}=\frac{3 E_{0} I}{L^{2}}\left[\begin{array}{cccccc}
0 & 0 & 0 & 0 & 0 & 0 \\
0 & 0 & -1 & 0 & 0 & 1 \\
0 & -1 & L & 0 & 1 & 0 \\
0 & 0 & 0 & 0 & 0 & 0 \\
0 & 0 & 1 & 0 & 0 & -1 \\
0 & 1 & 0 & 0 & -1 & -L
\end{array}\right], \\
\Delta \mathbf{K}_{3}^{(e)}=\frac{9 E_{0} I}{2 L^{3}}\left[\begin{array}{cccccc}
0 & 0 & 0 & 0 & 0 & 0 \\
0 & 4 & 2 L & 0 & -4 & 2 L \\
0 & 2 L & L^{2} & 0 & -2 L & L^{2} \\
0 & 0 & 0 & 0 & 0 & 0 \\
0 & -4 & -2 L & 0 & 4 & -2 L \\
0 & 2 L & L^{2} & 0 & -2 L & L^{2}
\end{array}\right]
\end{gathered}
$$

and $\Delta \mathbf{M}_{k}^{(e)}, k=1,2, \ldots, 7$, are deterministic matrices:

$$
\begin{aligned}
\Delta \mathbf{M}_{1}^{(e)}=\frac{\rho_{0} L}{8}\left[\begin{array}{cccccc}
1 & 0 & 0 & 1 & 0 & 0 \\
0 & 1 & \frac{L}{4} & 0 & 1 & -\frac{L}{4} \\
0 & \frac{L}{4} & \frac{L^{2}}{16} & 0 & \frac{L}{4} & -\frac{L^{2}}{16} \\
1 & 0 & 0 & 1 & 0 & 0 \\
0 & 1 & \frac{L}{4} & 0 & 1 & -\frac{L}{4} \\
0 & -\frac{L}{4} & -\frac{L^{2}}{16} & 0 & -\frac{L}{4} & \frac{L^{2}}{16}
\end{array}\right], \\
\mathbf{M M}_{2}^{(e)}=\frac{\rho_{0} L}{8}\left[\begin{array}{cccccc}
-2 & 0 & 0 & 0 & 0 & 0 \\
0 & -3 & -\frac{5 L}{8} & 0 & 0 & \frac{L}{8} \\
0 & -\frac{5 L}{8} & -\frac{L^{2}}{8} & 0 & \frac{L}{8} & \frac{L^{2}}{8} \\
0 & 0 & 0 & -2 & 0 & 0 \\
0 & 0 & \frac{L}{8} & 0 & 3 & -\frac{5 L}{8} \\
0 & \frac{L}{8} & 0 & 0 & -\frac{5 L}{8} & \frac{L^{2}}{8}
\end{array}\right],
\end{aligned}
$$




$$
\begin{aligned}
& \Delta \mathbf{M}_{3}^{(e)}=\frac{\rho_{0} L}{8}\left[\begin{array}{cccccc}
1 & 0 & 0 & -1 & 0 & 0 \\
0 & \frac{9}{4} & \frac{L}{8} & 0 & -\frac{9}{4} & \frac{L}{8} \\
0 & \frac{L}{8} & -\frac{L^{2}}{16} & 0 & -\frac{5 L}{8} & \frac{3 L^{2}}{16} \\
-1 & 0 & 0 & 1 & 0 & 0 \\
0 & -\frac{9}{4} & -\frac{5 L}{8} & 0 & \frac{9}{4} & -\frac{L}{8} \\
0 & \frac{5 L}{8} & \frac{3 L^{2}}{16} & 0 & -\frac{L}{8} & -\frac{L^{2}}{16}
\end{array}\right], \\
& \Delta \mathbf{M}_{4}^{(e)}=\frac{\rho_{0} L}{8}\left[\begin{array}{cccccc}
0 & 0 & 0 & 0 & 0 & 0 \\
0 & 1 & \frac{3 L}{4} & 0 & 0 & -\frac{L}{4} \\
0 & \frac{3 L}{4} & \frac{L^{2}}{4} & 0 & -\frac{L}{4} & 0 \\
0 & 0 & 0 & 0 & 0 & 0 \\
0 & 0 & -\frac{L}{8} & 0 & 0 & \frac{3 L}{4} \\
0 & -\frac{L}{4} & 0 & 0 & \frac{3 L}{4} & -\frac{L^{2}}{4}
\end{array}\right], \\
& \Delta \mathbf{M}_{5}^{(e)}=\frac{\rho_{0} L}{16}\left[\begin{array}{cccccc}
0 & 0 & 0 & 0 & 0 & 0 \\
0 & -3 & -L & 0 & 3 & -L \\
0 & -L & -\frac{L^{2}}{8} & 0 & L & -\frac{3 L^{2}}{8} \\
0 & 0 & 0 & 0 & 0 & 0 \\
0 & 3 & L & 0 & -3 & L \\
0 & -L & -\frac{3 L^{2}}{8} & 0 & L & -\frac{L^{2}}{8}
\end{array}\right], \\
& \Delta \mathbf{M}_{6}^{(e)}=\frac{\rho_{0} L^{2}}{64}\left[\begin{array}{cccccc}
0 & 0 & 0 & 0 & 0 & 0 \\
0 & 0 & -1 & 0 & 0 & 1 \\
0 & -1 & -L & 0 & 1 & 0 \\
0 & 0 & 0 & 0 & 0 & 0 \\
0 & 0 & 1 & 0 & 0 & -1 \\
0 & 1 & 0 & 0 & -1 & L
\end{array}\right] \text {, } \\
& \Delta \mathbf{M}_{7}^{(e)}=\frac{\rho_{0} L}{32}\left[\begin{array}{cccccc}
0 & 0 & 0 & 0 & 0 & 0 \\
0 & 1 & \frac{L}{2} & 0 & -1 & \frac{L}{2} \\
0 & \frac{L}{2} & \frac{L^{2}}{4} & 0 & -\frac{L}{2} & \frac{L^{2}}{4} \\
0 & 0 & 0 & 0 & 0 & 0 \\
0 & -1 & -\frac{L}{2} & 0 & 1 & -\frac{L}{2} \\
0 & \frac{L}{2} & \frac{L^{2}}{4} & 0 & -\frac{L}{2} & \frac{L^{2}}{4}
\end{array}\right]
\end{aligned}
$$

and $X_{k}^{(e)}, k=1,2,3$, and $Y_{k}^{(e)}, k=1,2, \ldots, 7$, are the stiffness matrix and mass matrix weighted integrals:

$$
\begin{aligned}
X_{k}^{(e)} & =\int_{-1}^{+1} \xi^{k-1} f(\xi) \mathrm{d} \xi, \quad k=1,2,3, \\
Y_{k}^{(e)} & =\int_{-1}^{+1} \xi^{k-1} g(\xi) \mathrm{d} \xi, \quad k=1,2, \ldots, 7,
\end{aligned}
$$

where $\xi$ is the local (natural) coordinate system for the beam element (e). The global matrices may be assembled from all of the element matrices using standard finite element methodology.

Using the finite element method, the eigenvalue problem is expressed for any type of finite element as:

$$
\begin{aligned}
\left(\mathbf{K}-\lambda_{j} \mathbf{M}\right) \phi_{j} & =\mathbf{0}, \\
\phi_{j}^{\mathrm{T}} \mathbf{M} \phi_{j} & =1,
\end{aligned}
$$


where $\mathbf{K}$ and $\mathbf{M}$ are the stochastic global stiffness and mass matrices, respectively, $\lambda_{j}$ is the $j$ th eigenvalue and $\phi_{j}$ is the $j$ th eigenvector. Because the stiffness and mass matrices are stochastic, then the $j$ th eigenvalue and the $j$ th eigenvector will be random. In Graham and Deodatis [9], a first-order perturbation approximation to the $j$ th eigenvalue $\lambda_{j}$ was found:

$$
\begin{aligned}
\lambda_{j} & =\lambda_{j 0}+\Delta \lambda_{j} \\
& \approx \lambda_{j 0}+\boldsymbol{\phi}_{j 0}^{\mathrm{T}}\left[\sum_{e=1}^{N}\left(\sum_{k=1}^{3} \Delta \mathbf{K}_{k}^{(e)} X_{k}^{(e)}-\lambda_{j 0} \sum_{n=1}^{7} \Delta \mathbf{M}_{n}^{(e)} Y_{n}^{(e)}\right)\right] \phi_{j 0},
\end{aligned}
$$

where $\lambda_{j 0}$ and $\phi_{j 0}$ are the deterministic parts of the $j$ th eigenvalue and the $j$ th eigenvector, which are calculated using the following expressions:

$$
\begin{aligned}
\left(\mathbf{K}_{0}-\lambda_{j 0} \mathbf{M}_{0}\right) \phi_{j 0} & =\mathbf{0}, \\
\phi_{j 0}^{\mathrm{T}} \mathbf{M}_{0} \phi_{j 0} & =1 .
\end{aligned}
$$

Fox and Kapoor [8] and Zhu and Wu [30] derived a first-order approximation to the $j$ th eigenvector as:

$$
\begin{aligned}
\phi_{j} \approx & \phi_{j 0}+\mathbf{C}_{j}^{-1}\left(-\Delta \mathbf{K} \phi_{j 0}+\lambda_{j 0} \Delta \mathbf{M} \phi_{j 0}+\Delta \lambda_{j} \mathbf{M}_{0} \phi_{j 0}-\frac{1}{2} \lambda_{j 0} \mathbf{M}_{0} \phi_{j 0} \phi_{j 0}^{\mathrm{T}} \Delta \mathbf{M} \phi_{j 0}\right) \\
\approx & \phi_{j 0}+\mathbf{C}_{j}^{-1} \sum_{e=1}^{N} \sum_{k=1}^{3}\left(-\Delta \mathbf{K}_{k}^{(e)} \phi_{j 0}+\phi_{j 0}^{\mathrm{T}} \Delta \mathbf{K}_{k}^{(e)} \phi_{j 0} \mathbf{M}_{0} \phi_{j 0}\right) \cdot X_{k}^{(e)} \\
& +\mathbf{C}_{j}^{-1} \sum_{e=1}^{N} \sum_{n=1}^{7}\left(\lambda_{j 0} \Delta \mathbf{M}_{n}^{(e)} \phi_{j 0}+\frac{3}{2} \lambda_{j 0} \boldsymbol{\phi}_{j 0}^{\mathrm{T}} \Delta \mathbf{M}_{n}^{(e)} \boldsymbol{\phi}_{j 0} \mathbf{M}_{0} \phi_{j 0}\right) \cdot Y_{k}^{(e)},
\end{aligned}
$$

where $\lambda_{j 0}$ and $\phi_{j 0}$ are calculated using Eq. (12), and $\mathbf{C}_{j}$ is a deterministic matrix:

$$
\mathbf{C}_{j}=\mathbf{K}_{0}-\lambda_{j 0} \mathbf{M}_{0}+\lambda_{j 0} \mathbf{M}_{0} \phi_{j 0} \phi_{j 0}^{\mathrm{T}} \mathbf{M}_{0}
$$

According to Clough and Penzien [4] the vector of the maximum deflections of a structure due to a deterministic earthquake loading is:

$$
\mathbf{U}_{\max }=\sqrt{\mathbf{U}_{1, \max }^{2}+\mathbf{U}_{2, \text { max }}^{2}+\cdots+\mathbf{U}_{N M D, \max }^{2}}
$$

where $N M D$ is the number of vibrational modes for the given structure. The vector $\mathbf{U}_{j, \max }, j=1,2, \ldots, N M D$, is the maximum deflection for a given mode number $j$ :

$$
\mathbf{U}_{j, \max }=\phi_{j} \cdot\left[\boldsymbol{\phi}_{j}^{\mathrm{T}} \cdot \mathbf{M} \cdot \mathbf{1}\right] S_{d}\left(\xi_{j}, \lambda_{j}\right)
$$

where $\lambda_{j}$ and $\phi_{j}$ are the $j$ th eigenvalue and the $j$ th eigenvector, respectively, $\mathbf{M}$ is the stochastic global mass matrix, 1 is a vector of 1's, $S_{d}\left(\xi_{j}, \lambda_{j}\right)$ is the displacement response spectrum for the given earthquake loading, and $\xi_{j}$ is the damping ratio for mode $j$. Because $\lambda_{j}, \phi_{j}$, and $\mathbf{M}$ are all random, the maximum deflection is also random. For the following analysis it is assumed that $\xi_{j}$ is deterministic and that it is small enough so that damping does not have a significant effect on the eigenvalues.

The random maximum deflection vector may be approximated using a first-order Taylor expansion of Eqs (15) and (16) around the mean values of the weighted integrals: 


$$
\begin{aligned}
\mathbf{U}_{\max } \approx & \mathbf{U}_{\max , 0}+\sum_{j=1}^{N M D} \sum_{e=1}^{N} \sum_{k=1}^{3}\left[\frac{\partial \mathbf{U}_{\max }}{\partial \mathbf{U}_{j, \max }} \cdot \frac{\partial \mathbf{U}_{j, \max }}{\partial X_{k}^{(e)}}\right]_{0} \cdot X_{k}^{(e)} \\
& +\sum_{j=1}^{N M D} \sum_{e=1}^{N} \sum_{n=1}^{7}\left[\frac{\partial \mathbf{U}_{\max }}{\partial \mathbf{U}_{j, \max }} \cdot \frac{\partial \mathbf{U}_{j, \max }}{\partial Y_{n}^{(e)}}\right]_{0} \cdot Y_{n}^{(e)},
\end{aligned}
$$

where the subscript 0 indicates that the quantity in brackets is taken at the (zero) mean value of the weighted integrals and $\mathbf{U}_{\max , 0}$ is the maximum deflection vector $\mathbf{U}_{\max }$ evaluated at the zero-mean values of the weighted integrals:

$$
\mathbf{U}_{\max , 0}=\left[\sum_{j=1}^{N}\left\{\mathbf{U}_{j, \max , 0}\right\}^{2}\right]^{1 / 2}=\left[\sum_{j=1}^{N}\left\{\phi_{j 0} \cdot\left(\phi_{j 0}^{\mathrm{T}} \cdot \mathbf{M}_{0} \cdot \mathbf{1}\right) \cdot S_{d}\left(\xi_{j}, \lambda_{j 0}\right)\right\}^{2}\right]^{1 / 2}
$$

Taking the partial derivatives of Eqs (11), (13), (15), and (16) and substituting into Eq. (17):

$$
\mathbf{U}_{\max } \approx \mathbf{U}_{\max , 0}+\sum_{j=1}^{N M D} \sum_{e=1}^{N}\left[\sum_{k=1}^{3} \boldsymbol{\Delta}_{X} \mathbf{U}_{\max , j k}^{(e)} \cdot X_{k}^{(e)}+\sum_{n=1}^{7} \boldsymbol{\Delta}_{Y} \mathbf{U}_{\max , j n}^{(e)} \cdot Y_{n}^{(e)}\right],
$$

where $\Delta_{X} \mathbf{U}_{\max , j k}^{(e)}, k=1,2,3$, and $\Delta_{Y} \mathbf{U}_{\max , j n}^{(e)}, n=1,2, \ldots, 7$, are deterministic vectors, defined as:

$$
\begin{aligned}
\boldsymbol{\Delta}_{X} \mathbf{U}_{\max , j k}^{(e)}= & \operatorname{diag}\left[\nabla \mathbf{U}_{j, \max }\right] \cdot\left[-\beta_{j k}^{(e)} \boldsymbol{\phi}_{j 0}+\varepsilon_{j k}^{(e)} \eta_{j} \boldsymbol{\phi}_{j 0}-\rho_{j} \mathbf{C}_{j}^{-1} \Delta \mathbf{K}_{k}^{(e)} \boldsymbol{\phi}_{j 0}\right. \\
& \left.+\varepsilon_{j k}^{(e)} \rho_{j} \mathbf{C}_{j}^{-1} \mathbf{M}_{0} \boldsymbol{\phi}_{j 0}+\varepsilon_{j k}^{(e)} \rho_{j} \boldsymbol{\phi}_{j 0} \frac{1}{S_{d}\left(\xi_{j}, \lambda_{j 0}\right)} \frac{\partial S_{d}}{\partial \lambda_{j}}\left(\xi_{j}, \lambda_{j 0}\right)\right] \\
& j=1,2, \ldots, N M D, k=1,2,3, e=1,2, \ldots, N \\
\boldsymbol{\Delta}_{Y} \mathbf{U}_{\max , j n}^{(e)}= & \operatorname{diag}\left[\nabla \mathbf{U}_{j, \max }\right] \cdot\left[\alpha_{j n}^{(e)} \boldsymbol{\phi}_{j 0}+\lambda_{j 0} \gamma_{j n}^{(e)} \boldsymbol{\phi}_{j 0}-\frac{3}{2} \lambda_{j 0} \eta_{j} \xi_{j n}^{(e)} \boldsymbol{\phi}_{j 0}\right. \\
& +\lambda_{j 0} \rho_{j} \mathbf{C}_{j}^{-1} \Delta \mathbf{M}_{n}^{(e)} \boldsymbol{\phi}_{j 0}-\frac{3}{2} \lambda_{j 0} \xi_{j n}^{(e)} \rho_{j} \mathbf{C}_{j}^{-1} \mathbf{M}_{0} \boldsymbol{\phi}_{j 0} \\
& \left.-\lambda_{j 0} \xi_{j n}^{(e)} \rho_{j} \boldsymbol{\phi}_{j 0} \frac{1}{S_{d}\left(\xi_{j}, \lambda_{j 0}\right)} \frac{\partial S_{d}}{\partial \lambda_{j}}\left(\xi_{j}, \lambda_{j 0}\right)\right] \\
& j=1,2, \ldots, N M D, n=1,2, \ldots, 7, e=1,2, \ldots, N
\end{aligned}
$$

where diag[ ] represents a diagonal matrix whose diagonal components consist of the vector within parentheses, and the $i$ th component of the vector $\nabla \mathbf{U}_{j \text {, max }}$ is defined as:

$$
\left[\nabla \mathbf{U}_{j, \max }\right]_{i}=\frac{\left[\mathbf{U}_{j, \max , 0}\right]_{i}}{\left[\mathbf{U}_{\max , 0}\right]_{i}}
$$

The constants $\alpha_{j k}^{(e)}, \gamma_{j k}^{(e)}, \xi_{j k}^{(e)}, k=1,2, \ldots, 7, \beta_{j k}^{(e)}, \varepsilon_{j k}^{(e)}, k=1,2,3, \eta_{j}$, and $\rho_{j}$ are closed-form quantities, given as:

$$
\begin{aligned}
\alpha_{j k}^{(e)} & =\phi_{j 0}^{\mathrm{T}} \Delta \mathbf{M}_{k}^{(e)} \mathbf{1} S_{d}\left(\xi_{j}, \lambda_{j 0}\right), \\
\beta_{j k}^{(e)} & =\phi_{j 0}^{\mathrm{T}} \Delta \mathbf{K}_{k}^{(e)} \mathbf{C}_{j}^{-1} \mathbf{M}_{0} \mathbf{1} S_{d}\left(\xi_{j}, \lambda_{j 0}\right),
\end{aligned}
$$




$$
\begin{aligned}
\gamma_{j k}^{(e)} & =\phi_{j 0}^{\mathrm{T}} \Delta \mathbf{M}_{k}^{(e)} \mathbf{C}_{j}^{-1} \mathbf{M}_{0} \mathbf{1} S_{d}\left(\xi_{j}, \lambda_{j 0}\right), \\
\varepsilon_{j k}^{(e)} & =\boldsymbol{\phi}_{j 0}^{\mathrm{T}} \Delta \mathbf{K}_{k}^{(e)} \boldsymbol{\phi}_{j 0}, \\
\xi_{j k}^{(e)} & =\boldsymbol{\phi}_{j 0}^{\mathrm{T}} \Delta \mathbf{M}_{k}^{(e)} \boldsymbol{\phi}_{j 0}, \\
\eta_{j} & =\boldsymbol{\phi}_{j 0}^{\mathrm{T}} \mathbf{M}_{0} \mathbf{C}_{j}^{-1} \mathbf{M}_{0} \mathbf{1} S_{d}\left(\xi_{j}, \lambda_{j 0}\right), \\
\rho_{j} & =\boldsymbol{\phi}_{j 0}^{\mathrm{T}} \mathbf{M}_{0} \mathbf{1} S_{d}\left(\xi_{j}, \lambda_{j 0}\right) .
\end{aligned}
$$

Using Eq. (19), the mean and variance of the maximum deflection vector are estimated as:

$$
\begin{aligned}
\mathcal{E}\left[\mathbf{U}_{\max }\right]= & \mathbf{U}_{\max , 0}, \\
\operatorname{Var}\left[\mathbf{U}_{\max }\right]= & \sum_{j_{1}=1}^{N M D} \sum_{j_{2}=1}^{N M D} \sum_{e_{1}=1}^{N} \sum_{e_{2}=1}^{N} \sum_{k=1}^{3} \sum_{n=1}^{3} \operatorname{diag}\left(\boldsymbol{\Delta}_{X} \mathbf{U}_{\max , j_{1} k}^{\left(e_{1}\right)}\right) \cdot \boldsymbol{\Delta}_{X} \mathbf{U}_{\max , j_{2} n}^{\left(e_{2}\right)} \mathcal{E}\left[X_{k}^{\left(e_{1}\right)} X_{n}^{\left(e_{2}\right)}\right] \\
& +\sum_{j_{1}=1}^{N M D} \sum_{j_{2}=1}^{N M D} \sum_{e_{1}=1}^{N} \sum_{e_{2}=1}^{N} \sum_{k=1}^{7} \sum_{n=1}^{7} \operatorname{diag}\left(\boldsymbol{\Delta}_{Y} \mathbf{U}_{\max , j_{1} k}^{\left(e_{1}\right)}\right) \cdot \boldsymbol{\Delta}_{Y} \mathbf{U}_{\max , j_{2} n}^{\left(e_{2}\right)} \mathcal{E}\left[Y_{k}^{\left(e_{1}\right)} Y_{n}^{\left(e_{2}\right)}\right] \\
& +2 \sum_{j_{1}=1}^{N M D} \sum_{j_{2}=1}^{N M D} \sum_{e_{1}=1}^{N} \sum_{e_{2}=1}^{N} \sum_{k=1}^{3} \sum_{n=1}^{7} \operatorname{diag}\left(\boldsymbol{\Delta}_{X} \mathbf{U}_{\max , j_{1} k}^{\left(e_{1}\right)}\right) \cdot \boldsymbol{\Delta}_{Y} \mathbf{U}_{\max , j_{2} n}^{\left(e_{2}\right)} \mathcal{E}\left[X_{k}^{\left(e_{1}\right)} Y_{n}^{\left(e_{2}\right)}\right]
\end{aligned}
$$

\section{Variability response functions}

Using the expressions for the weighted integrals given in Eqs (8) and (9), the specific form of the weighted integral covariances can be calculated for beam-column elements. Following a method similar to that used in Deodatis and Graham [7], the variance of the maximum deflection vector for a structure discretized using the beam-column elements described earlier is written as:

$$
\begin{aligned}
\operatorname{Var}\left[\mathbf{U}_{\max }\right]= & \int_{-\infty}^{\infty} S_{f f}(\kappa) \mathbf{V} \mathbf{R} \mathbf{F}_{1}(\kappa) \mathrm{d} \kappa+\int_{-\infty}^{\infty} S_{g g}(\kappa) \mathbf{V} \mathbf{R} \mathbf{F}_{2}(\kappa) \mathrm{d} \kappa \\
& +\int_{-\infty}^{\infty} C_{f g}(\kappa) \mathbf{V R F}_{3}(\kappa) \mathrm{d} \kappa+\int_{-\infty}^{\infty} D_{f g}(\kappa) \mathbf{V R F}_{4}(\kappa) \mathrm{d} \kappa,
\end{aligned}
$$

where $S_{f f}(\kappa)$ and $S_{g g}(\kappa)$ are the power spectral density functions describing the elastic modulus and the mass density, and $C_{f g}(\kappa)$ (the co-spectrum) is the real part and $D_{f g}(\kappa)$ (the quad-spectrum) is the imaginary part of the cross spectral density functions describing the cross-correlation between the elastic modulus and the mass density (Bendat and Piersol [1]):

$$
S_{f g}(\kappa)=C_{f g}(\kappa)-\mathrm{i} \cdot D_{f g}(\kappa) .
$$

The $\mathbf{V R F}_{i}(\kappa), i=1,2,3,4$, are the variability response functions, given in closed form as:

$$
\begin{aligned}
\mathbf{V R F}_{1}(\kappa)= & \sum_{j_{1}=1}^{N M D} \sum_{j_{2}=1}^{N M D} \sum_{e_{1}=1}^{N} \sum_{e_{2}=1}^{N} \sum_{k=1}^{3} \sum_{n=1}^{3} \operatorname{diag}\left(\Delta_{X} \mathbf{U}_{\text {max } j_{1} k}^{\left(e_{2}\right)}\right) \cdot \Delta_{X} \mathbf{U}_{\max , j_{2} n}^{\left(e_{2}\right)} \\
& \times\left[\left(Q_{e_{1} k} Q_{e_{2} n}+W_{e_{1} k} W_{e_{2} n}\right) \cos \left(\Delta x_{e_{2} e_{1}} \kappa\right)-\left(W_{e_{1} k} Q_{e_{2} n}-Q_{e_{1} k} W_{e_{2} n}\right) \sin \left(\Delta x_{e_{2} e_{1}} \kappa\right)\right],
\end{aligned}
$$




$$
\begin{aligned}
\mathbf{V R F}_{2}(\kappa)= & \sum_{j_{1}=1}^{N M D} \sum_{j_{2}=1}^{N M D} \sum_{e_{1}=1}^{N} \sum_{e_{2}=1}^{N} \sum_{k=1}^{7} \sum_{n=1}^{7} \operatorname{diag}\left(\boldsymbol{\Delta}_{Y} \mathbf{U}_{\max , j_{1} k}^{(e)}\right) \cdot \Delta_{Y} \mathbf{U}_{\max , j_{2} n}^{(e)} \\
& \times\left[\left(Q_{e_{1} k} Q_{e_{2} n}+W_{e_{1} k} W_{e_{2} n}\right) \cos \left(\Delta x_{e_{2} e_{1}} \kappa\right)-\left(W_{e_{1} k} Q_{e_{2} n}-Q_{e_{1} k} W_{e_{2} n}\right) \sin \left(\Delta x_{e_{2} e_{1}} \kappa\right)\right], \\
\mathbf{V R F}_{3}(\kappa)= & 2 \sum_{j_{1}=1}^{N M D} \sum_{j_{2}=1}^{N M D} \sum_{e_{1}=1}^{N} \sum_{e_{2}=1}^{N} \sum_{k=1}^{3} \sum_{n=1}^{7} \operatorname{diag}\left(\Delta_{X} \mathbf{U}_{\max , j_{1} k}^{\left(e_{2}\right)}\right) \cdot \Delta_{Y} \mathbf{U}_{\max , j_{2} n}^{(e)} \\
& \times\left[\left(Q_{e_{1} k} Q_{e_{2} n}+W_{e_{1} k} W_{e_{2} n}\right) \cos \left(\Delta x_{e_{2} e_{1}} \kappa\right)-\left(W_{e_{1} k} Q_{e_{2} n}-Q_{e_{1} k} W_{e_{2} n}\right) \sin \left(\Delta x_{e_{2} e_{1}} \kappa\right)\right], \\
\mathbf{V R F}_{4}(\kappa)= & 2 \sum_{j_{1}=1}^{N M D} \sum_{j_{2}=1}^{N M D} \sum_{e_{1}=1}^{N} \sum_{e_{2}=1}^{N} \sum_{k=1}^{3} \sum_{n=1}^{7} \operatorname{diag}\left(\Delta_{X} \mathbf{U}_{\max , j_{1} k}^{\left(e_{2}\right)}\right) \cdot \Delta_{Y} \mathbf{U}_{\max , j_{2} n}^{(e)} \\
& \times\left[\left(Q_{e_{1} k} Q_{e_{2} n}+W_{e_{1} k} W_{e_{2} n}\right) \sin \left(\Delta x_{e_{2} e_{1}} \kappa\right)+\left(W_{e_{1} k} Q_{e_{2} n}-Q_{e_{1} k} W_{e_{2} n}\right) \cos \left(\Delta x_{e_{2} e_{1}} \kappa\right)\right],
\end{aligned}
$$

where the matrices $\Delta K_{k}^{(e)}, k=1,2,3, e=1,2, \ldots, N$, are given in Eq. (6), $\Delta \mathbf{M}_{n}^{(e)}, n=1,2, \ldots, 7, e=1,2$, $\ldots, N$, are given in Eq. (7), $\boldsymbol{\Delta}_{X} \mathbf{U}_{\max , j_{2} n}^{\left(e_{2}\right)}, j=1,2, \ldots, N M D, k=1,2,3$, is defined in Eq. (20), $\boldsymbol{\Delta}_{Y} \mathbf{U}_{\max , j n}^{(e)}, j=$ $1,2, \ldots, N M D, n=1,2, \ldots, 7$, is defined in Eq. (21), $\Delta x_{e_{2} e_{1}}$ is the distance between the centerpoints of elements $\left(e_{1}\right)$ and $\left(e_{2}\right)$, and the $Q$ 's and $W$ 's are the following closed-form expressions:

$$
\begin{aligned}
Q_{e 1}= & \frac{4}{\kappa L_{e}} \sin \left(\frac{\kappa L_{e}}{2}\right), \quad e=1,2, \ldots, N, \\
Q_{e 3}= & \frac{4}{\kappa^{3} L_{e}^{3}}\left(\left(\kappa^{2} L_{e}^{2}-8\right) \sin \left(\frac{\kappa L_{e}}{2}\right)+4 \kappa L_{e} \cos \left(\frac{\kappa L_{e}}{2}\right)\right), \quad e=1,2, \ldots, N, \\
Q_{e 5}= & \frac{4}{\kappa^{5} L_{e}^{5}}\left(\left(384-48 \kappa^{2} L_{e}^{2}+\kappa^{4} L_{e}^{4}\right) \sin \left(\frac{\kappa L_{e}}{2}\right)+8 \kappa L_{e}\left(\kappa^{2} L_{e}^{2}-24\right) \cos \left(\frac{\kappa L_{e}}{2}\right)\right), \\
e= & 1,2, \ldots, N, \\
Q_{e 7}= & \frac{4}{\kappa^{7} L_{e}^{7}}\left(\left(-46080+5760 \kappa^{2} L_{e}^{2}-120 \kappa^{4} L_{e}^{4}+\kappa^{6} L_{e}^{6}\right) \sin \left(\frac{\kappa L_{e}}{2}\right)\right. \\
& \left.+12 \kappa L_{e}\left(1920-80 \kappa^{2} L_{e}^{2}+\kappa^{4} L_{e}^{4}\right) \cos \left(\frac{\kappa L_{e}}{2}\right)\right), \quad e=1,2, \ldots, N, \\
W_{e 2}= & \frac{4}{\kappa^{2} L_{e}^{2}}\left(-2 \sin \left(\frac{\kappa L_{e}}{2}\right)+\kappa^{2} L_{e}^{2} \cos \left(\frac{\kappa L_{e}}{2}\right)\right), \quad e=1,2, \ldots, N, \\
W_{e 4}= & \frac{4}{\kappa^{4} L_{e}^{4}}\left(6\left(8-\kappa^{2} L_{e}^{2}\right) \sin \left(\frac{\kappa L_{e}}{2}\right)-\kappa L_{e}\left(24-\kappa^{2} L_{e}^{2}\right) \cos \left(\frac{\kappa L_{e}}{2}\right)\right), \quad e=1,2, \ldots, N, \\
W_{e 6}= & \frac{4}{\kappa^{6} L_{e}^{6}}\left(\kappa L_{e}\left(1920-80 \kappa^{2} L_{e}^{2}+\kappa^{4} L_{e}^{4}\right) \cos \left(\frac{\kappa L_{e}}{2}\right)\right. \\
& \left.-10\left(384-48 \kappa^{2} L_{e}^{2}+\kappa^{4} L_{e}^{4}\right) \sin \left(\frac{\kappa L_{e}}{2}\right)\right), \quad e=1,2, \ldots, N .
\end{aligned}
$$

In these expressions, $e$ is an element number and $Q_{e 2}=Q_{e 4}=Q_{e 6}=W_{e 1}=W_{e 3}=W_{e 5}=W_{e 7}=0$.

It is worth noting that numerous first-order approximations are used in the formulation of the variability response functions; in the numerical results Monte Carlo simulations of the weighted integrals will be used to estimate the error inherent in these first-order approximations. 


\section{Spectral-distribution-free upper bounds of displacement variability}

The establishment of upper bounds on the displacement variability from Eq. (26) is somewhat complicated, because of the terms involving the cross-spectral density function. Assuming that the quad-spectrum $D_{f g}(\kappa)$ vanishes, which is realistic for the consideration of material properties, an upper bound estimate for the variance of displacement $U_{i}$ is found as:

$$
\operatorname{Var}\left[U_{i}\right] \leqslant \sigma_{f f}^{2} V R F_{1 i}\left(\kappa^{*}\right)+\sigma_{g g}^{2} V R F_{2 i}\left(\kappa^{*}\right)+\gamma_{f g} \sigma_{f f} \sigma_{g g} V R F_{3 i}\left(\kappa^{*}\right),
$$

where:

$$
\begin{aligned}
& \sigma_{f f}^{2} V R F_{1 i}\left(\kappa^{*}\right)+\sigma_{g g}^{2} V R F_{2 i}\left(\kappa^{*}\right)+\gamma_{f g} \sigma_{f f} \sigma_{g g} V R F_{3 i}\left(\kappa^{*}\right) \\
& \quad \geqslant \sigma_{f f}^{2} V R F_{1 i}(\kappa)+\sigma_{g g}^{2} V R F_{2 i}(\kappa)+\gamma_{f g} \sigma_{f f} \sigma_{g g} V R F_{3 i}(\kappa), \quad-\infty \leqslant \kappa \leqslant \infty .
\end{aligned}
$$

$\gamma_{f g}$ is defined here as $\sigma_{f g}^{2} / \sigma_{f f} \sigma_{g g}$ (the cross-correlation coefficient, which ranges from 0 to 1 ). $V R F_{j i}$ is the $i$ th component of vector $\mathbf{V R F} \mathbf{F}_{j}, j=1,2,3,4$, and $\sigma_{f f}^{2}$ and $\sigma_{g g}^{2}$ are the variances of stochastic fields $f(x)$ and $g(x)$, respectively.

\section{Numerical examples}

\subsection{Portal frame}

The maximum horizontal deflection $\left(H_{\max }\right)$ of the upper right corner of the reinforced concrete portal frame shown in Fig. 1, having 16 nodes and 15 elements, is considered in this numerical example. Unless otherwise indicated, the coefficients of variation of the elastic modulus $\left(\sigma_{f f}\right)$ and the mass density $\left(\sigma_{g g}\right)$ are assumed to 0.10 . For demonstration purposes, a $0.5 \mathrm{~g}$ earthquake loading that follows the response spectrum given for Soil Type 2 in Fig. 2 (Uniform Building Code [27]) is used. For this particular portal frame, all modes other than the first vibrational mode yield negligible contributions to the maximum deflection vector; therefore, only the first eigenvalue will be considered (i.e., $N M D=1$ ). Note that the mean value of $H_{\max }$ is found to be $1.39 \mathrm{~cm}$.

The variability response functions (Eq. (28)) are plotted as a function of $\kappa$ in Fig. 3. It is interesting to note that for smaller $\kappa, V R F_{2}$, which corresponds to the mass density autocorrelation, dominates; however, $V R F_{1}$, which corresponds to the elastic modulus autocorrelation, is dominant for higher $\kappa$. In other words, small-scale random fluctuations in mass density have little effect on the maximum displacement variability relative to the effects of small-scale fluctuations in the elastic modulus. Note that the variability response function $V R F_{4}$ for this maximum deflection problem is very close to zero for all $\kappa$.

The spectral-distribution-free estimate on the upper bound of $\operatorname{COV}\left(H_{\max }\right)$ given in Eq. (30) is provided in Fig. 4 for the portal frame as a function of the cross-correlation parameter $\gamma_{f g}$. The maximum upper bound estimate on the coefficient of variation of $H_{\max }$ is $\operatorname{COV}\left(H_{\max }\right)=0.141$, which corresponds to the case when the random fields $f(x)$ and $g(x)$ are uncorrelated $\left(\gamma_{f g}=0\right)$.

The upper bounds calculated above are very significant for engineering applications, as they depend only on the mean and variance of the stochastic fields describing elastic modulus and mass density. The variability response function technique is also applicable if the spectral density functions describing these properties are known or can be assumed. Solely for demonstration purposes in the following example, it is assumed that the auto-spectral density functions which characterize the stochastic fields $f(x)$ and $g(x)$ (associated with the stochastic elastic modulus and the stochastic mass density) are:

$$
\begin{aligned}
& S_{f f}(\kappa)=\frac{2}{\sqrt{\pi}} \sigma_{f f}^{2} d_{E}^{3} \kappa^{2} \mathrm{e}^{-d_{E}^{2} \kappa^{2}}, \\
& S_{g g}(\kappa)=\frac{2}{\sqrt{\pi}} \sigma_{g g}^{2} d_{\rho}^{3} \kappa^{2} \mathrm{e}^{-d_{\rho}^{2} \kappa^{2}},
\end{aligned}
$$




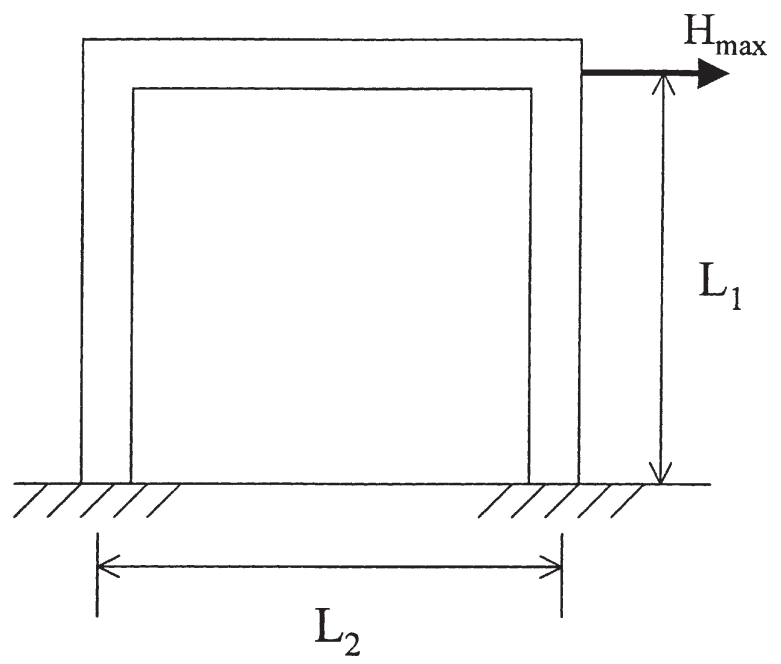

Fig. 1. Reinforced concrete portal frame considered in numerical example. $L_{1}=L_{2}=L=5 \mathrm{~m}, A=0.09 \mathrm{~m}^{2}, I=0,00068 \mathrm{~m}^{4}, E_{0}=20 \mathrm{GPa}$, $\rho_{0}=2400 \mathrm{~kg} / \mathrm{m}^{3}$.

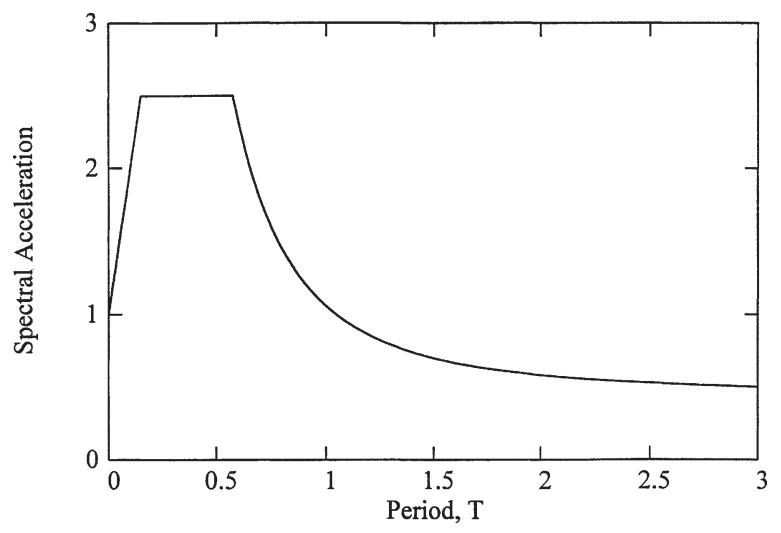

Fig. 2. Response spectrum used in numerical example (uniform building code [27]).

where $\sigma_{f f}$ and $\sigma_{g g}$ are the standard deviations of stochastic fields $f(x)$ and $g(x)$ (assumed to be 0.10 unless otherwise indicated), and $d_{E}$ and $d_{\rho}$ are parameters associated with the correlation distances of the stochastic fields $f(x)$ and $g(x)$. These auto-spectral density functions correspond to the autocorrelation functions:

$$
\begin{aligned}
& R_{f f}(\zeta)=\sigma_{f f}^{2}\left[1-\frac{\zeta^{2}}{2 d_{E}^{2}}\right] \mathrm{e}^{-\zeta^{2} / 4 d_{E}^{2}}, \\
& R_{g g}(\zeta)=\sigma_{g g}^{2}\left[1-\frac{\zeta^{2}}{2 d_{\rho}^{2}}\right] \mathrm{e}^{-\zeta^{2} / 4 d_{\rho}^{2}},
\end{aligned}
$$

where $\zeta$ is a separation distance. Eq. (27) indicated that the cross-spectral density function is the combination of a real, even co-spectrum $\left(C_{f g}(\kappa)\right)$ and an imaginary, odd quad-spectrum (i $\left.\cdot D_{f g}(\kappa)\right)$. It is reasonable to assume that for the crosscorrelation of material properties, the quad-spectrum is zero $\left(D_{f g}(\kappa)=0\right)$. For the purposes of this numerical example, it is assumed that the cross-spectral density function takes the form:

$$
S_{f g}\left(\kappa_{x}\right)=\gamma_{f g} \sqrt{S_{f f}\left(\kappa_{x}\right) S_{g g}\left(\kappa_{x}\right)}=\gamma_{f g} \frac{2}{\sqrt{\pi}} \sigma_{f f} \sigma_{g g} d_{E}^{3 / 2} d_{\rho}^{3 / 2} \kappa_{x}^{2} \mathrm{e}^{-\left(d_{E}^{2}+d_{\rho}^{2}\right) \kappa_{x}^{2} / 2},
$$




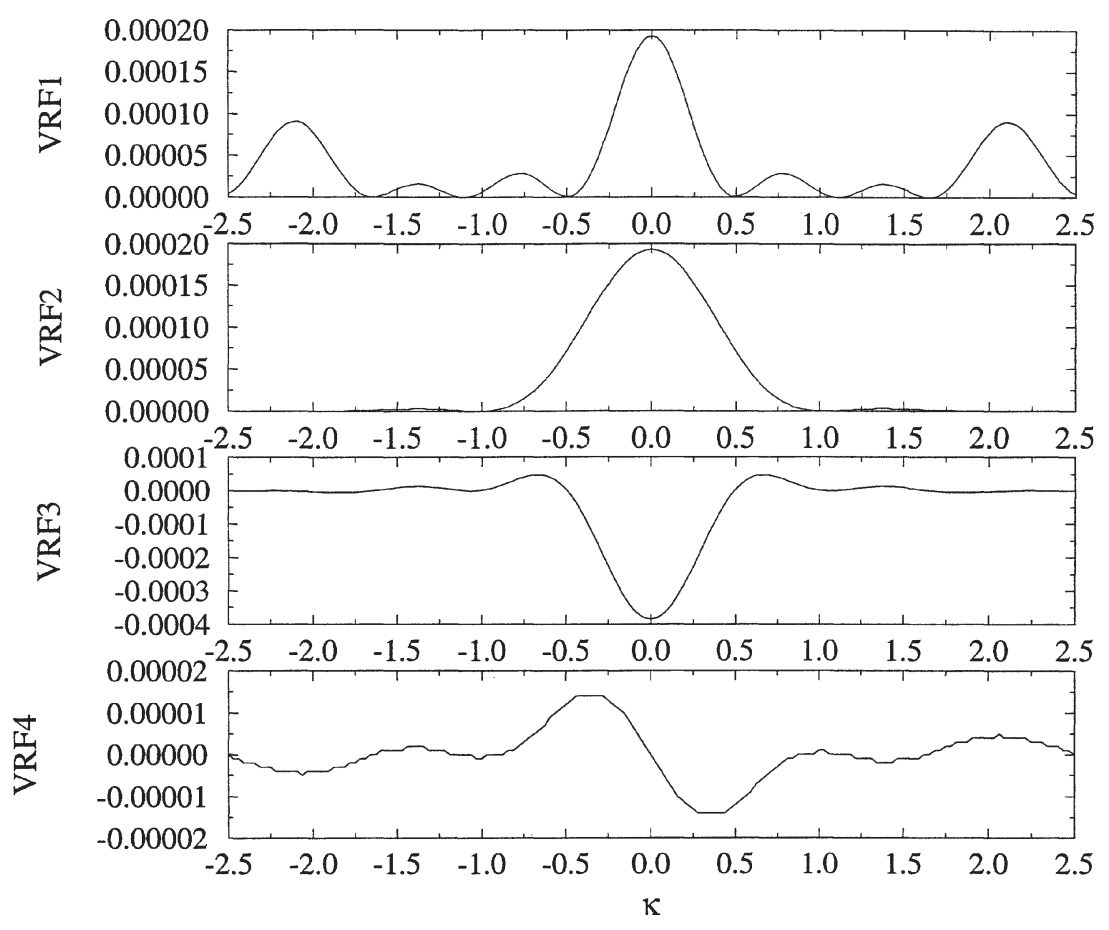

Fig. 3. Variability response functions for maximum deflection of the portal frame $\left(H_{\max }\right)$ as a function of $\kappa$.

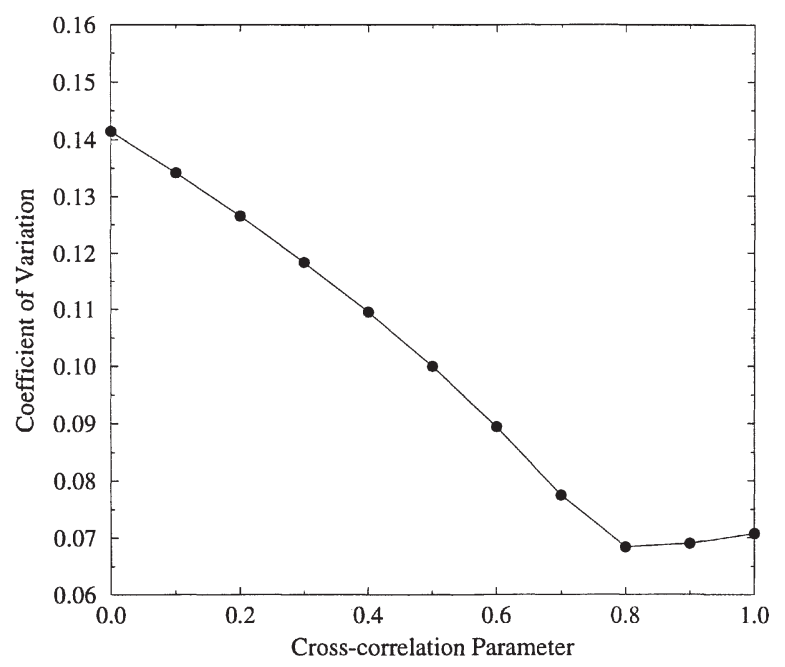

Fig. 4. Estimates on the upper bounds of the coefficient of variation of $H_{\max }$ as a function of cross-correlation parameter $\gamma_{f g}$.

where $\gamma_{f g}$ is the cross-correlation coefficient between the two random fields $f(x)$ and $g(x)$. This cross-spectral density function corresponds to a cross-correlation function:

$$
R_{f g}(\zeta)=2 \sqrt{2} \gamma_{f g} \sigma_{f f} \sigma_{g g}\left[\frac{d_{\rho} d_{E}}{d_{\rho}^{2}+d_{E}^{2}}\right]^{3 / 2}\left[1-\frac{\zeta^{2}}{d_{\rho}^{2}+d_{E}^{2}}\right] \mathrm{e}^{-\zeta^{2} / 2\left(d_{\rho}^{2}+d_{E}^{2}\right)}
$$

The coefficient of variation $(\mathrm{COV})$ of the maximum deflection $\left(H_{\max }\right)$ is calculated by numerically performing the integrals in Eq. (26), for different values of the correlation distance parameter $d=d_{E}=d_{\rho}$. The results are plotted 


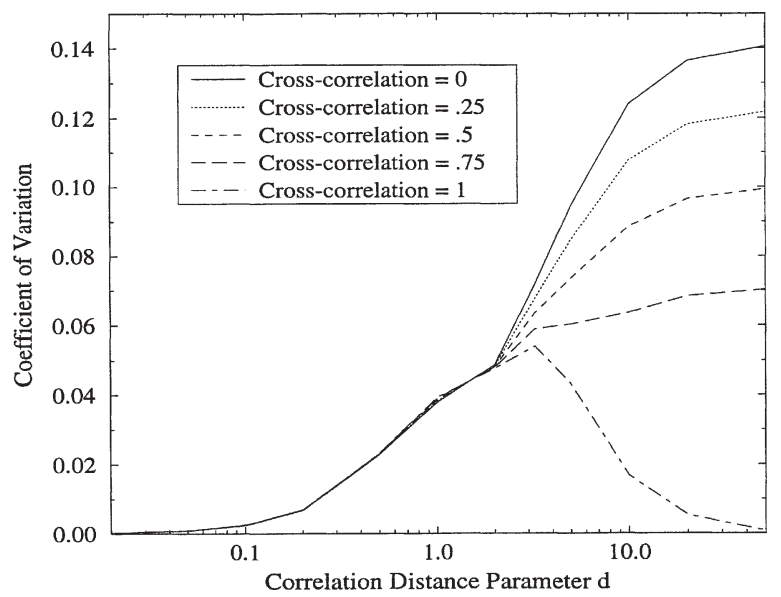

Fig. 5. Coefficient of variation of $H_{\max }$ of the portal frame as a function of correlation distance parameter $d=d_{E}=d_{\rho}$ for various cross-correlation parameters $\gamma_{f g}$.

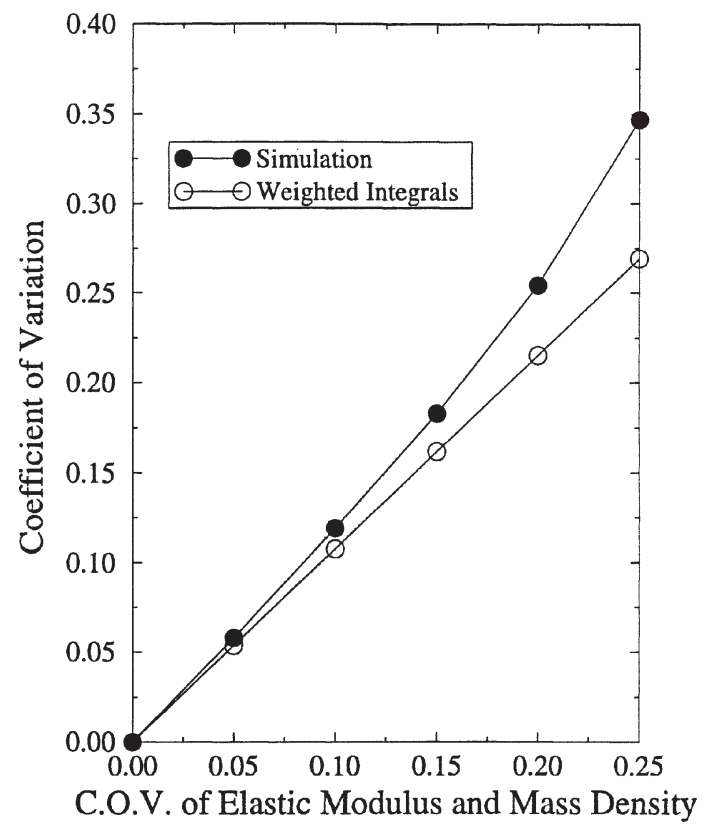

Fig. 6. Coefficient of variation of $H_{\max }$ of the portal frame as a function of $\sigma_{f f}=\sigma_{g g}$ for correlation distance parameter $d=d_{E}=d_{\rho}=10$ and cross-correlation parameter $\gamma_{f g}=0.25$.

in Fig. 5 for various cross-correlation parameters $\gamma_{f g}$. The cross-correlation parameter has a significant effect on these results, especially as $d$ becomes large.

As mentioned earlier, there are first-order approximations used in the variability response function formulations (see Eq. (17)). Figure 6 provides a comparison between the results using the weighted integral method and Monte Carlo simulation techniques as a function of the standard deviations of the stochastic $f(x)$ and $g(x)$. As the values of the coefficient of variation of the elastic modulus and the mass density become larger, larger discrepancies are expected between the weighted integral-based and Monte Carlo simulation-based results. Figure 6 shows this behavior for the case where the correlation distance parameter $d_{E}=d_{\rho}=10$ and the cross-correlation parameter $\gamma_{f g}=0.25$. Similar behavior is exhibited for all values of the correlation distance parameters and the cross-correlation parameter. 


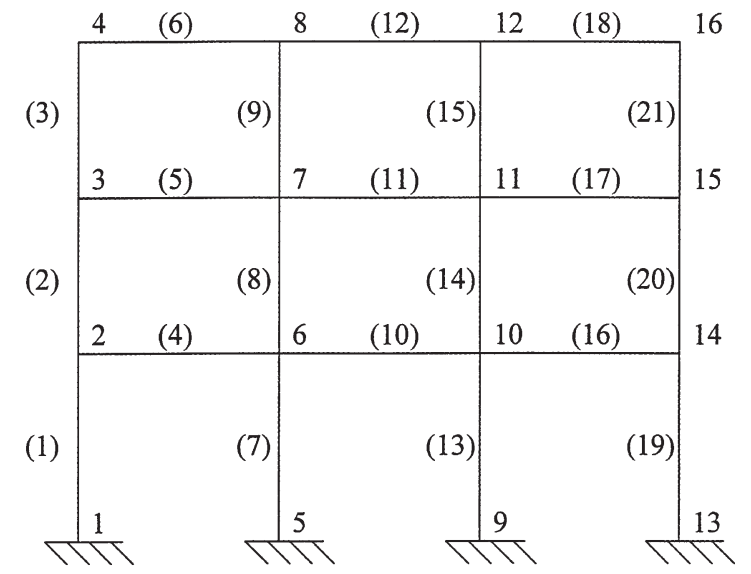

Elements (1),(7),(13),(19) - .55m x .55m cross-section

Elements (2),(8),(14),(20) - .5m x .5m cross-section

Elements (3),(9),(15),(21) - .45m x.45m cross-section

Fig. 7. Three-story, three-bay frame.

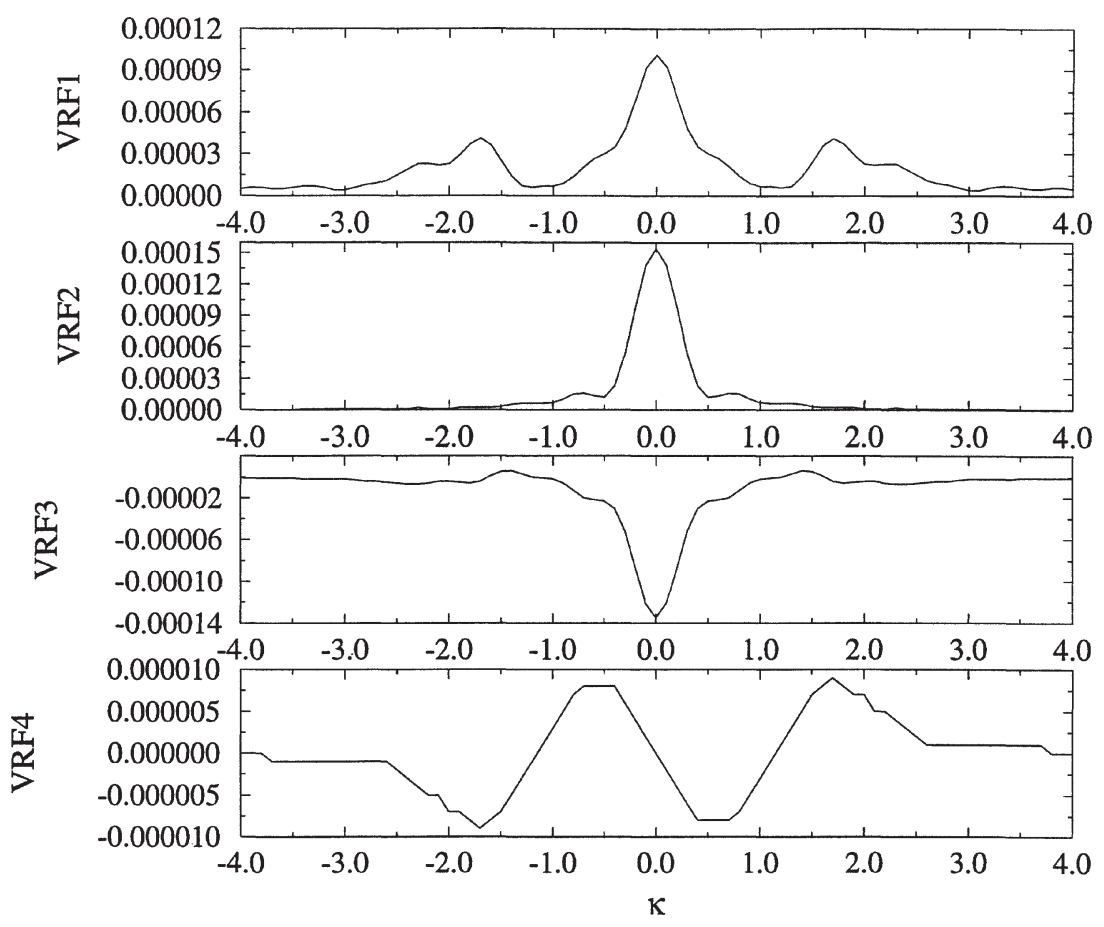

Fig. 8. Variability response function for maximum horizontal deflection of node 16 as a function of $\kappa_{x}$.

\subsection{Three-story three-bay reinforced concrete frame}

The maximum horizontal deflection $\left(U_{16, \max }\right)$ of Node 16 of the reinforced concrete frame shown in Fig. 7, having 16 nodes and 21 elements, is considered in this numerical example. It is assumed that the stochastic fields describing the elastic modulus and the mass density are independent for all column elements, and that there is only correlation between beam elements on the same level. This assumption is reasonable when considering the construction proce- 


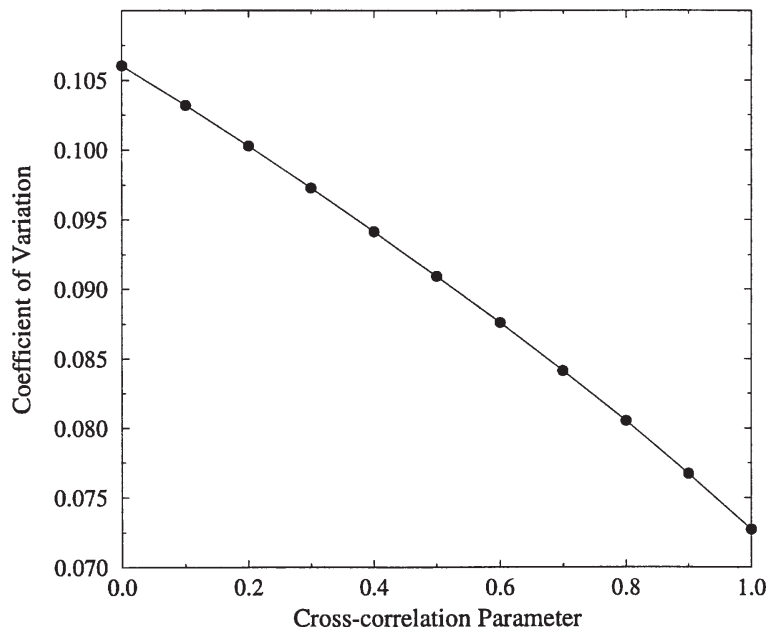

Fig. 9. Estimates on the upper bounds of the coefficient of variation of the deflection of node 16 as a function of cross-correlation parameter $\gamma_{f g}$.

dure for reinforced concrete frames. Unless otherwise indicated, the coefficients of variation of the elastic modulus $\left(\sigma_{f f}\right)$ and the mass density $\left(\sigma_{g g}\right)$ are assumed to 0.10 . The response spectrum given in Fig. 2 (Uniform Building Code [27]) is used for demonstration purposes. For this particular portal frame, all modes other than the first and second vibrational modes yield negligible contributions to the maximum deflection vector; therefore, only the first and second eigenvalues and eigenvectors will be considered (i.e., $N M D=2$ ). Note that the mean value of $U_{16 \text {,max }}$ is $1.51 \mathrm{~cm}$.

The weighted integral based variability response functions (Eq. (28)) are plotted as a function of $\kappa_{x}$ in Fig. 8 . It is interesting to note that similar to the portal frame results, $V R F_{2}$, which corresponds to the mass density autocorrelation, dominates for smaller $\kappa_{x}$; however, $V R F_{1}$, which corresponds to the elastic modulus autocorrelation, is dominant for higher $\kappa_{x}$. Figure 8 also shows that the variability response function $V R F_{4}$ for this maximum deflection problem is very close to zero for all $\kappa_{x}$.

The spectral-distribution-free estimate on the upper bound of $\operatorname{COV}\left(U_{16, \max }\right)$ given in Eq. (30) is provided in Fig. 9 for $U_{16, \max }$ as a function of the cross-correlation parameter $\gamma_{f g}$. The maximum upper bound estimate on the coefficient of variation of $U_{16 \text {,max }}$ is $\operatorname{COV}\left(U_{16, \text { max }}\right)=0.106$, which corresponds to the case when the random fields $f(x)$ and $g(x)$ are uncorrelated $\left(\gamma_{f g}=0\right)$.

\section{Conclusions}

Variability response functions have been successfully formulated which consider randomness in the maximum deflection of a structure under design earthquake loading. The numerical examples show that randomness in the material properties can have a significant effect on the variability of this maximum deflection. Therefore, the safety margin that is generally assumed in formulating the earthquake design response spectrum may be reduced by randomness in the structural parameters. The variability response functions also allow estimates of the spectral-distributionfree upper bounds on the maximum deflection variability which depend only on the mean and variances of the stochastic fields describing the elastic modulus and the mass density.

\section{Acknowledgements}

The author wishes to thank Professor George Deodatis for his insightful comments about this work. 


\section{References}

[1] J.S. Bendat and A.G. Piersol, Random Data: Analysis and Measurement Procedures, Wiley, 1986.

[2] W.E. Boyce, Random eigenvalue problems, in: Probabilistic Methods in Applied Mathematics, Part 1, A.T. Bharucha-Reid, ed., Academic Press, New York, 1968, pp. 1-73.

[3] W.E. Boyce and N.M. Xia, The approach to normality of the solutions of random boundary and eigenvalue problems with weakly correlated coefficients, Quarterly of Applied Mathematics 38 (1983), 419-445.

[4] R.W. Clough and J. Penzien, Dynamics of Structures, McGraw-Hill, 1975.

[5] J.D. Collins and W.T. Thomson, The eigenvalue problem for structural systems with statistical properties, AIAA Journal 7(4) (1969), 642648.

[6] G. Deodatis, Bounds on response variability of stochastic finite element systems, J. Engineering Mechanics, ASCE 116(3) (1990), 565-585.

[7] G. Deodatis and L. Graham, The weighted integral method and the variability response function as part of a SFEM formulation, in: Uncertainty Modeling in Finite Element, Fatigue \& Stability of Structures, World Scientific, 1997, pp. 71-116.

[8] R.L. Fox and M.P. Kapoor, Rates of change of eigenvalues and eigenvectors, AIAA Journal 6(12) (1968), $2426-2429$.

[9] L. Graham and G. Deodatis, Response and eigenvalue analysis of stochastic finite element systems with multiple correlated material and geometric properties, Probabilistic Engineering Mechanics (1998) (To appear).

[10] M. Grigoriu, A solution of the random eigenvalue problem by crossing theory, J. Sound and Vibration 58(1) (1992), 69-80.

[11] T.K. Hasselman and G.C. Hart, Modal analysis of random structural systems, J. Engineering Mechanics Division, ASCE 98 (1972), 561579.

[12] M. Hoshiya and H.C. Shah, Free vibration of stochastic beam-column, J. Engineering Mechanics Division, ASCE 97 (1971), $1239-1255$.

[13] W.H. Huang, Vibration of some structures with periodic random parameters, AIAA Journal 20(7) (1982), 1001-1008.

[14] R.A. Ibrahim, Structural dynamics with parameter uncertainties, Applied Mechanics Reviews, ASME 40(3) (1987), 309-328.

[15] R.N. Iyengar and C.S. Manohar, Probability distribution of the eigenvalues of the random string equation, J. Applied Mechanics, ASME 56 (1989), 202-207.

[16] H.U. Koyluoglu, Stochastic response and reliability analyses of structures with random properties subject to stationary random excitation, Ph.D. thesis, Princeton University, Princeton, NJ, 1995.

[17] C.S. Manohar and R.N. Iyengar, Probability distribution of the eigenvalues of systems governed by the stochastic wave equation, Probabilistic Engineering Mechanics 8 (1993), 57-64.

[18] T. Nagashima and T. Tsutsumi, Stochastic eigenvalue analysis by SFEM Code 'SAINT', in: Computational Stochastic Mechanics, P.D. Spanos and C.A. Brebbia, eds, Computational Mechanics Publications, Southampton, co-published by Elsevier Applied Science, London, 1991, pp. 827-838.

[19] S. Nakagiri, H. Takabatake and S. Tani, Uncertain eigenvalue analysis of composite laminated plates by the stochastic finite element method, in: Advances in Aerospace Structural Analysis, ASME Aerospace Division AD-09, O.H. Burnside and C.H. Parr, eds, 1985, pp. 1-7.

[20] W. Purkert and J. Vom Scheidt, Stochastic eigenvalue problems for differential equations, Reports on Mathematical Physics 15(2) (1979), 205-227.

[21] S.A. Ramu and R. Ganesan, Response and stability of a stochastic beam-column using stochastic FEM, Computers \& Structures 54(2) (1995), 207-221.

[22] S.A. Ramu, R. Ganesan and T.S. Sankar, Stability analysis of stochastically parametered nonconservative columns, Int. J. Solids Structures 29(23) (1992), 2973-2988.

[23] M. Shinozuka and C.J. Astill, Random eigenvalue problems in structural analysis, AIAA Journal 10(4) (1972), 456-462.

[24] T.T. Soong and J.L. Bogdanoff, On the natural frequencies of a disordered linear chain of $N$ degrees of freedom, Int. J. Mech. Sci. 5(3) (1963), 237-265.

[25] P.D. Spanos and B.A. Zeldin, Galerkin sampling method for stochastic mechanics problems, J. Engineering Mechanics, ASCE 120(5) (1994), 1091-1106.

[26] T. Takada, Weighted integral method in stochastic finite element analysis, Probabilistic Engineering Mechanics 5(3) (1990), 146-156.

[27] Uniform building code, structural engineering design provisions, in: Int. Conf. of Building Officials, Whittier, CA, 1994.

[28] R. Vaicaitis, Free vibrations of beams with random characteristics, J. Sound and Vibration 35(1) (1974), $13-21$.

[29] Z. Zhang and S. Chen, The standard deviations of the eigensolutions for random MDOF systems, Computers \& Structures 39(6) (1991), $603-607$.

[30] W.Q. Zhu and W.Q. Wu, A stochastic finite element method for real eigenvalue problems, Probabilistic Engineering Mechanics 6(2):3,4 (1991), 228-232. 

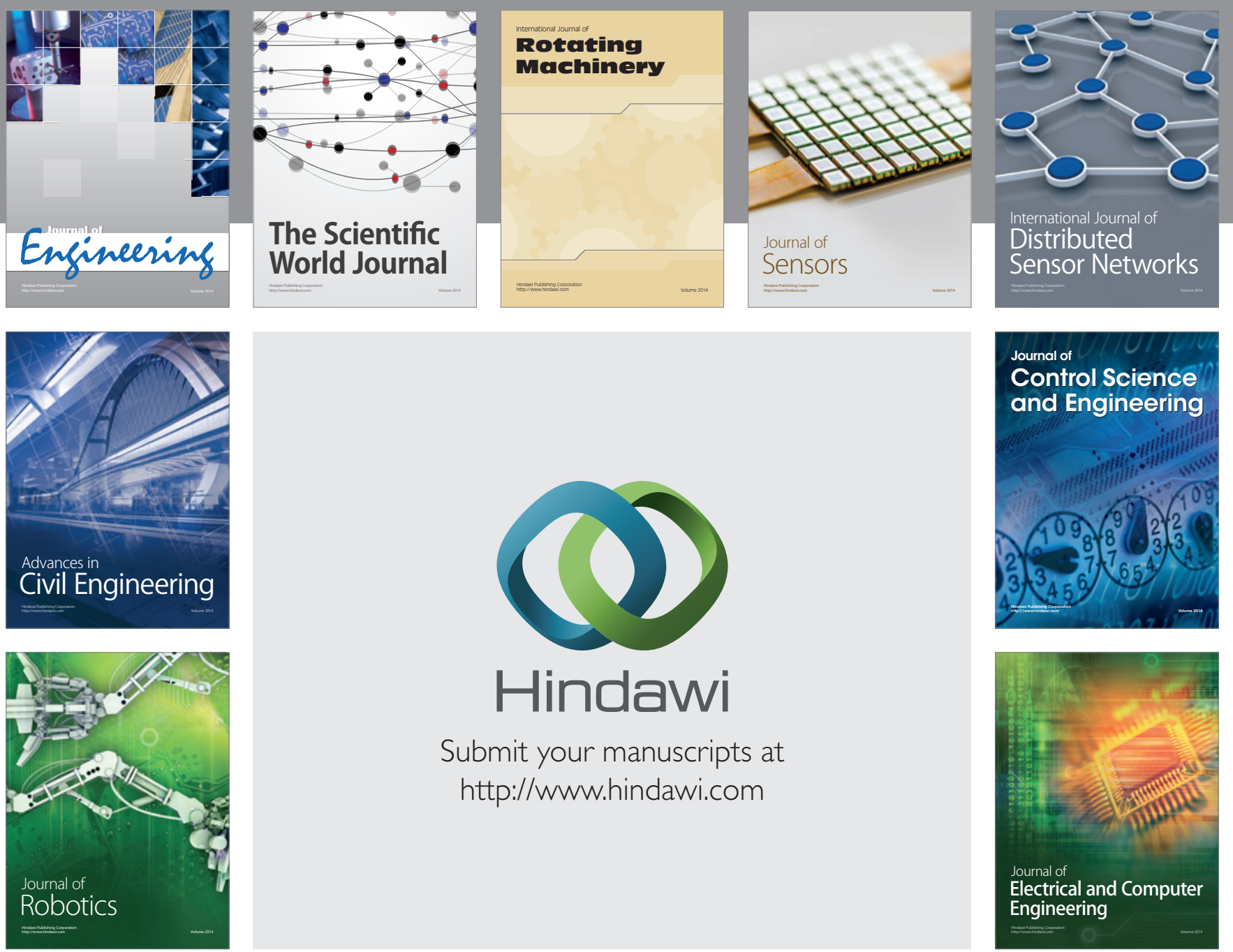

Submit your manuscripts at

http://www.hindawi.com
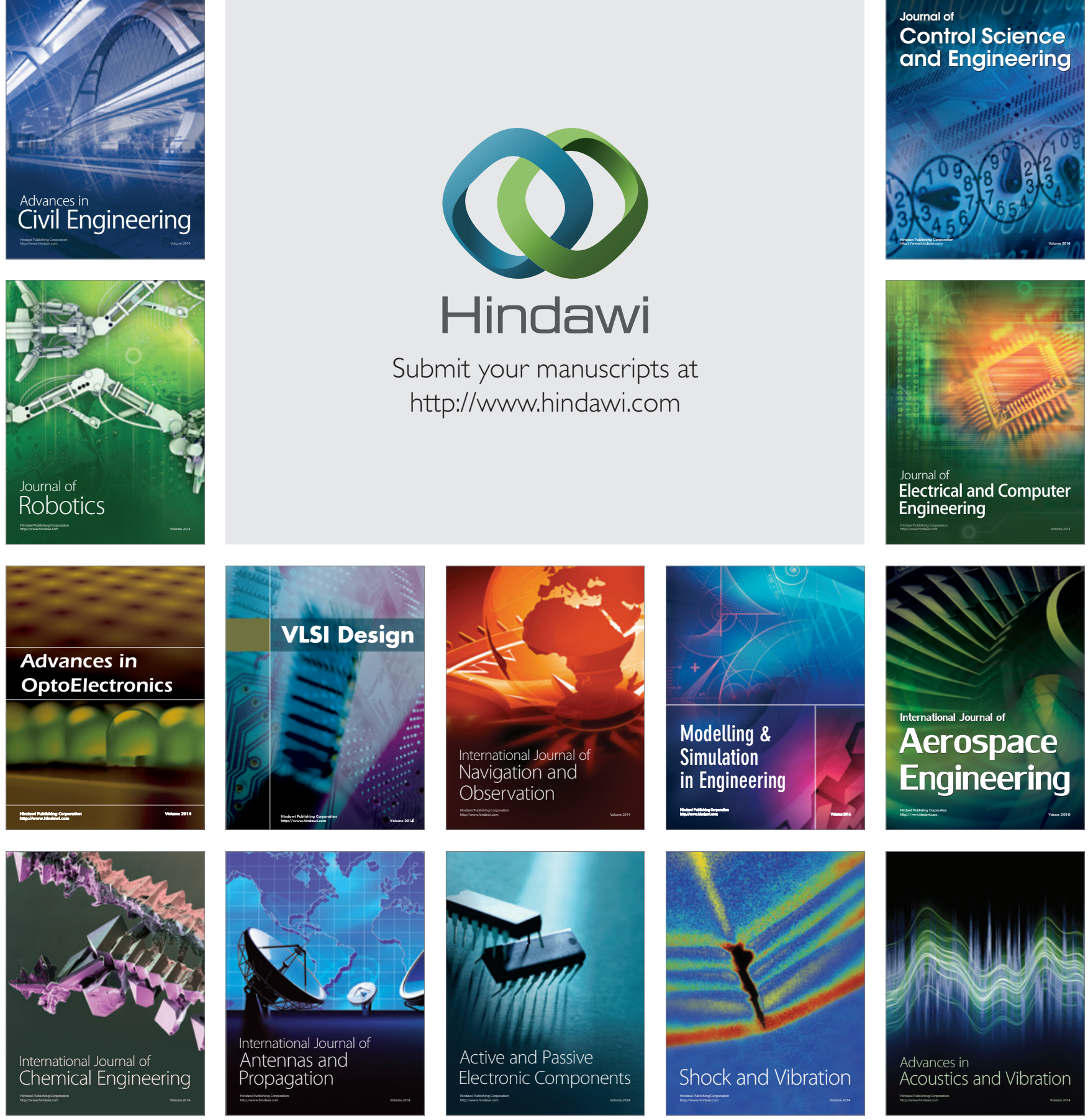